\title{
Factors Influencing Adherence to Antiretroviral Therapy among HIV Positive Adolescents at Adult Infectious Diseases Center in Lusaka, Zambia
}

\author{
Harrison C. Namoomba, Marjory Kabinga Makukula, Maureen M. Masumo \\ Department of Basic and Clinical Nursing Sciences, University of Zambia, Lusaka, Zambia \\ Email: harrisonamoomba@gmail.com
}

How to cite this paper: Namoomba, H.C., Makukula, M.K. and Masumo, M.M. (2019) Factors Influencing Adherence to Antiretroviral Therapy among HIV Positive Adolescents at Adult Infectious Diseases Center in Lusaka, Zambia. Open Journal of Nursing, 9, 458-480.

https://doi.org/10.4236/ojn.2019.94040

Received: February 19, 2019

Accepted: April 25, 2019

Published: April 28, 2019

Copyright $\odot 2019$ by author(s) and Scientific Research Publishing Inc. This work is licensed under the Creative Commons Attribution International License (CC BY 4.0).

http://creativecommons.org/licenses/by/4.0/

Open Access

\begin{abstract}
Background: Non-adherence to combination antiretroviral therapy leads to drug resistance, treatment failure and increased morbidity and mortality among HIV positive adolescents. The aim of this study was to assess factors influencing adherence to combination antiretroviral therapy among HIV positive adolescents at Adult Infectious Disease Center in Lusaka, Zambia. Methodology: A descriptive quantitative cross sectional study was conducted on 173 adolescent respondents on antiretroviral therapy who were selected using a systematic random sampling method. Data was collected using the questionnaires with the adopted Morisky drug adherence scale and analyzed using Statistical Package for the Social Sciences software, version 20. Chi square, fishers' exact statistical tests and the binary logistic regression model were used to determine true predictors and to adjust confounders of adherence. The cut off point for statistical significance was set at 5\%. Results: This study established that 76 (43.9\%) of the respondents were non-adherent to their antiretroviral therapy. Knowledge of HIV and disease progression was reported to be low but had no statistical impact on antiretroviral therapy adherence. Stigmatization was high $(99(57.2 \%))$ but did not significantly influence adherence to combination antiretroviral therapy on the study participants. Factors which were found to be statistically significant to adherence to therapy when a binary logistic regression was performed were experiencing side effects to therapy ( $\mathrm{p}$-value 0.047 , odds ratio $=0.412$ ); understanding reason for taking combination antiretroviral therapy ( $\mathrm{p}$-value 0.006 , odds ratio $=$ 5.978 ) and being reminded to take drugs ( $p$-value 0.006 , odds ratio $=0.505$ ). Conclusion: The study found that there was high level of non-adherence to combination antiretroviral therapy which could subsequently lead to in-
\end{abstract}


creased treatment failure. More studies on factors influencing non-adherence to combination antiretroviral therapy need to be conducted to develop evidence-based practice model for HIV positive adolescents care.

\section{Keywords}

Adherence, Antiretroviral Drugs, Knowledge

\section{Introduction}

HIV and AIDS have continued to affect young people despite significant scientific advances in prevention and treatment [1]. In 2015, 1.4 million (80\%) adolescents living with HIV globally were in Sub-Saharan Africa [2]. The Zambia Demographic Health Survey showed that about $4.4 \%$ of the adolescents aged 15 19 years in Zambia had HIV infection [3]. According to one study [4], about 4000 children were initiated on cART from the University Teaching Hospital, Pediatrics and Adult Infectious Diseases Centers program. Evidence has shown that HIV positive adolescents could have acquired the infection perinatally or in infancy through breastfeeding [2] [5]. Some literature indicates that the majority of adolescents acquired HIV through mother to child transmission (MTCT) and about $18 \%$ got infected later in their lives [6]. The WHO treatment guidelines recommended combination antiretroviral therapy (cART) initiation to all HIV infected adolescents to prevent HIV-related morbidity and mortality [7]. Some evidence has shown that access to cART to treat HIV infection and AIDS in resource-poor countries had improved over the past several years [8]. The cART when taken correctly could lead to sustained viral suppression and reduction in occurrence of AIDS-defining and non-AIDS-defining complications. In order to achieve sustained viral suppression and clinical benefits, WHO further emphasized strategies such as: early diagnosis and treatment, adherence to cART counseling and viral load monitoring among others to all HIV positive adolescents [9].

Adolescents diagnosed with HIV are started on cART to achieve complete viral suppression and prevention of occurrence of treatment failure later in their life [5] [10]. Other authors have stated that incomplete viral suppression could lead to treatment failure especially among the adolescents [9] [11]. To achieve complete viral suppression and prevention of treatment failure, the WHO guidelines recommended first, second and third line streams of cART from which treatment for the HIV positive adolescents could be commenced [9]. First line cART regimen is initiated by providing an optimized, fixed-dose cART regimen of Tenofovir (TDF) + Lamivudine/Emtricitabine (XTC) + Efavirenz (EFV)/Nevirapine (NVP) or Abacavir (ABC) + XTC + EFV (weight based dosing) for adolescents.

Once treatment is commenced, enhanced adherence to cART for individual HIV positive adolescent patients have been emphasized [7] [12]. According to evidence adherence has been strongly correlated with HIV viral suppression, reduced rates of drug resistance, enhanced survival and improved life expectancy 
among HIV positive adolescents [13].

The routine viral load monitoring is recommended as the most accurate objective available measure to determine effective cART response [7] [14]. Detectable viral load exceeding 1000 copies $/ \mathrm{ml}$ on two consecutive measurements within a three-month interval, with enhanced adherence support between measurements after at least six months of using first line cART, the adolescent would be said to have developed Virologic treatment failure. Virologic failure requires that the patients are switched to second line treatment [12]. Lopinavir boosted by ritonavir (LPV-r) is primarily the recommended second line choice regimen for HIV positive adolescents. Provision of third line cART occurs in very rare circumstances and is beyond the scope of most cART providers [5] [12].

Despite the emphasis on measures to prevent AIDS-defining complications and optimal cART provision, some studies have reviewed that non-adherence to cART among adolescents has remained prevalent [2] [5] [14] [15] [16] [17] [18]. Other authors have linked non-adherence to cART among adolescents to persistently elevated viral loads and progression of HIV infection into AIDS defining illnesses [19] [20]. While reasons for non-adherence to cART are not clear, several factors have been implicated and these include low level of knowledge about HIV, social cultural beliefs such as lack of social support and stigmatization among others. It has been reported that some social cultural beliefs impact negatively on conversional drugs adherence which could result in poor treatment outcomes [21]. Snyder found that lack of social support for HIV positive adolescents from the caregivers, friends, or clinicians could lead to non-adherence to cART resulting in virologic treatment failure [22]. In other reports, the overall level of comprehensive correct knowledge of HIV and AIDS remains very low among older adolescents aged 15 - 19 [23]. However some studies found that HIV-infected adolescents demonstrated good knowledge regarding HIV/AIDS and cART, including the consequences of developing drug resistance with suboptimal cART adherence [24]. Despite some reports that HIV positive adolescents have good knowledge regarding HIV/AIDS progression, non-adherence to cART and treatment failure have remained prevalent in this age group. Non-adherence to cART have been linked to treatment failure which defeats the primary goals of cART provision of maximal and durable suppression of viral load, restoration and preservation of immunologic function, improvement of quality of life, and reduction of HIV-related morbidity and mortality [5] [12]. The required level of 95\% cART drug adherence leads to viral suppression when ARV drugs are used as prescribed, and for life [5]. Non-adherence to cART causes transmutations of the virus and later drug resistance [6]. The transmutations of the HIV and drugs resistance leads to switching of regime to second or third line therapy, which rapidly exhaust the cART available options resulting in AIDS defining morbidities and mortality among HIV positive adolescents [15]. To prevent cART failure, intrapersonal and interpersonal related factors which could be linked to non-adherence to cART among adolescents have been investigated. 


\section{Materials and Methods}

\subsection{Materials}

The factors influencing adherence among HIV positive adolescents were investigated from the Social Ecological Model, (SEM) theoretical perspective of Individual, Interpersonal, Organizational/Institutional, and Community Levels. The SEM theoretical perspective stresses that individual behavior is influenced by factors at different levels as classified by Sallis and Owen [25]. The study was conducted between August and December 2018.

The study defined Non-adherence to cART as being unable to answer correctly all the responses on the 4 item Morisky drug compliance scale within the past one month from the date of data collection.

In this study, when the respondent correctly answered 12 or more questions on knowledge it was regarded as having high level of knowledge. The respondent was said to have low level of knowledge if they answered 1 to 11 questions on knowledge correctly.

Social support was defined as adequate when the respondent answered correctly 5 questions and above on the social support section. The respondents who had inadequate social support were those who only managed to correctly answer between 1 and 4 questions correctly.

Stigmatization was regarded as high when the respondent had 1 or 2 scores and as low with the score of 3 or 4 on the stigmatization section. Responses from the questionnaires were assigned numerical codes for entry and analysis using the SPSS software computer package version 20. Pearson's Chi square and fisher's exact statistical tests were used to determine the association between dependent (adherence to cART) and independent variables (knowledge of HIV and disease progression, social support, stigmatization and cultural beliefs). The fisher's exact test was used where the criteria for using the Pearson's chi square was not met. The binary logistic regression test was used to come up with the true predictors and to adjust confounders of adherence to cART among HIV positive adolescents. The cut off point for statistical significance was set at 5\%.

\subsection{Methods}

The study used a descriptive quantitative cross sectional research design. The design was selected because data was collected at one point and no interventions or manipulations were carried out on both the environment and the respondents.

The study population consisted of HIV positive adolescents on cART treatment receiving care at Adult Infectious Diseases Centre, Lusaka. The study targeted males and females adolescents aged between 16 and 19 years on cART for at least three months enrolled for care at Adult Infectious Diseases Centre. These were adolescents who had transitioned from children to adult hospital soon after attaining their $16^{\text {th }}$ birthday. Parents and guardians after this hospital transition seem to reduce on accompanying these HIV positive adolescents for the clinical 
follow ups.

A probability sampling method was adopted using systematic sampling method. The $\mathrm{k}^{\text {th }}$ subject on a scheduled booking was asked to participate in the study using $\mathrm{N} / \mathrm{n}$. Therefore, every $5^{\text {th }}$ subject of HIV positive adolescents on cART who was coming for scheduled visits was requested to participate in the study [26]. This method provided all the subjects in the population with equal chance to be included in the study. A sample size is a small fraction of the population to represent the target population under study [26]. The sample size was calculated according to Krejcie and Morgan formula for the limited or finite population [27]. The final sample size was 173 . The questionnaire with the adopted Morisky 4 item scale was used to determine level of adherence to cART among the eligible study participants. The validity and reliability of the instrument was insured by pretesting the instrument and which was also reviewed by team of experts.

\section{Ethical and Cultural Considerations}

The study was conducted in accordance with provisions of the Helsinki declaration (World Medical Association (WMA)), and privacy and confidentiality were safeguarded consistent with guidelines for research involving young people [28] [29].

A continuous evaluation of the research by the supervisor and the co-supervisor, and an ethical clearance (Ref: 006-06-18) to conduct the study was obtained from the University of Zambia School Of Medicine Biomedical Research Ethics Committee and National Health Research Council of Zambia. Written permissions were obtained from the School of Nursing Sciences, District Health Management Team (DHMT) and The UTHs Management.

\section{Results}

This paper presents data collected from $173 \mathrm{HIV}$ positive adolescents on cART aged between 16 and 19 years from Adult Infectious Diseases Center, Lusaka, Zambia.

\subsection{Social Demographic}

Almost half $49.1 \%$ (85) of the study respondents were males. Slightly above half $53.2 \%$ (92) of the respondents were within the age group range of $16-17$ years.

Slightly above half $52.6 \%$ (91) of the respondents had attained secondary school education. The majority $99.4 \%$ (172) of the respondents were living with their family i.e. biological parents, grandparents and uncle or aunty. Above one third 38.1\% (66) respondents had both biological parents still alive. The majority of the respondents had either their father $24.9 \%$ (43) or mother $17.9 \%$ (31) dead and $19.1 \%$ (33) had reported that both of their parents had died.

\subsection{Adherence Assessment}

Generally in this study $44 \%$ (76) of the respondents were found to be non adhe- 
rent to cART (Figure 1).

\subsection{Knowledge about HIV and Disease Progression}

Most respondents 59.5\% (103) were found to have low level knowledge about HIV and disease progression (Figure 2).

\subsection{Social Support for the Respondents}

The majority of the respondents $94.8 \%$ (164) reported to have adequate social support and only 5.2\% (9) had inadequate social support (Figure 3).
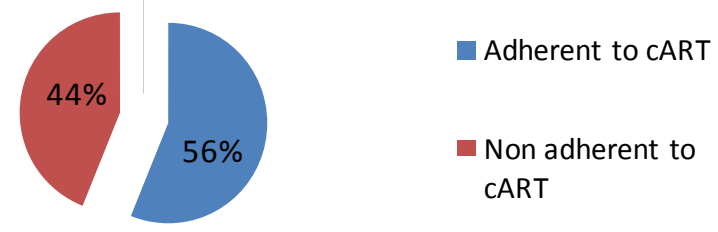

Figure 1. Adherence to cART assessment $(\mathrm{n}=173)$.

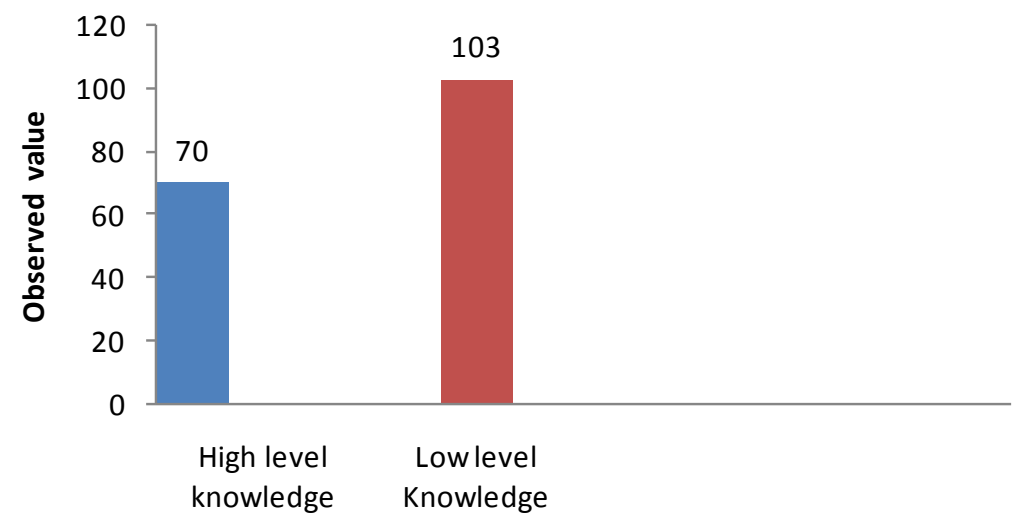

Level of knowledge of HIV and disease progression

Figure 2. Respondents level of knowledge about HIV and disease progression $(n=173)$.

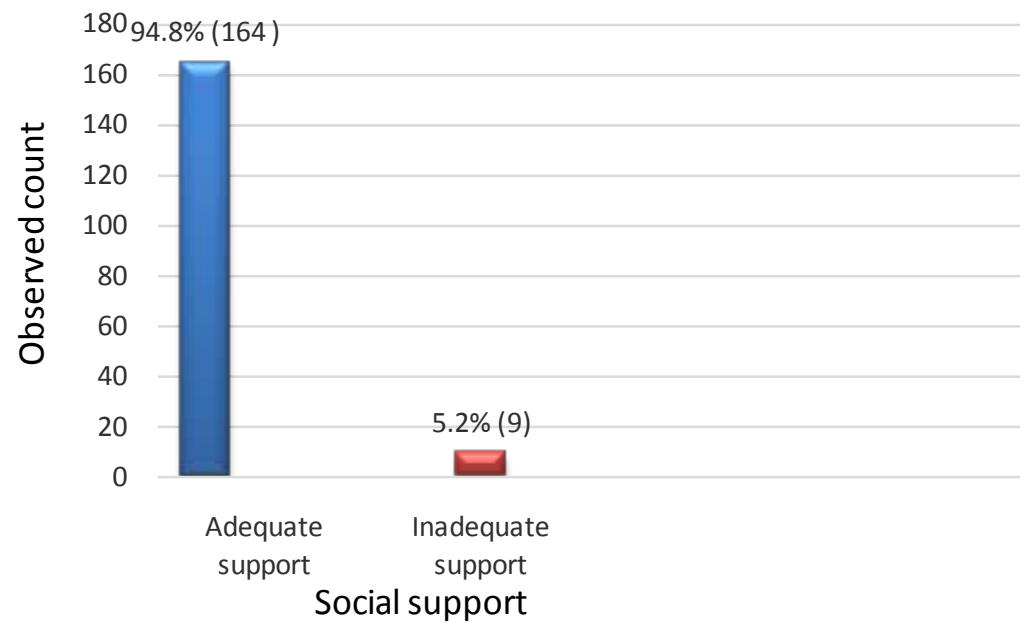

Figure 3. Level of social support for respondents $(n=173)$. 


\subsubsection{Religious Beliefs that Could Lead to Non-Adherence to cART}

All the participant 100\% (173) interviewed were from Christian community.

Out of 173 respondents $52.1 \%$ (90) believed that prayers and anointing oil could cure HIV/AIDS. Consequently 9\% (15) respondents reported that they had at one point abandoned cART in preference of prayers.

\subsubsection{Stigmatization Experience for the Respondents}

Generally $57.2 \%$ (99) of the respondents reported high level of stigmatization experience.

\subsubsection{Relationships among Study Variables}

This study established that $58 \%$ (56) of the respondents who were adherent to cART had low level knowledge. Among those that were reported to be non adherent, $62 \%$ (47) had low knowledge of HIV and disease progression. There was no significant association between adherence to cART and the level of knowledge ( $\mathrm{p}$-value was 0.585) (Table 1).

\section{1) Relationships between adherence to cART and the study variables}

The report indicates that $97 \%$ (94) respondents among those that were adherent to CART had adequate social support. It was further reported that $92 \%$ (70) of those who where non adherent had adequate social support. When social support was associated with adherence to cART among HIV positive adolescents it was found that there was no association (p-value 0.158). Overly about $95 \%$ (164) of the participants had demonstrated adequate social support.

Table 1. Association between adherence to cART and the study variables.

\begin{tabular}{ccccc}
\hline \multicolumn{5}{c}{ Adherence to cART } \\
\hline Parameter & Adherent & Non Adherent & Total & p-Value \\
\hline Knowledge level & & & & \\
High & $41(58.6 \%)$ & $29(41.4 \%)$ & $70(100.0 \%)$ & \\
Low & $56(54.4 \%)$ & $47(45.6 \%)$ & $103(100.0 \%)$ & \\
Total & $97(56.1 \%)$ & $76(43.9 \%)$ & $173(100.0 \%)$ & 0.585 \\
Social support level & & & & \\
Adequate & $94(57.3 \%)$ & $70(42.7 \%)$ & $164(100 \%)$ & \\
Inadequate & $3(33.3 \%)$ & $6(66.7 \%)$ & $9(100 \%)$ & \\
Total & $97(56.1 \%)$ & $76(43.9 \%)$ & $173(100 \%)$ & 0.158 \\
Religious belief & & & & \\
Positive & $86(55.8 \%)$ & $68(44.2 \%)$ & $154(100 \%)$ & \\
Negative & $11(57.9 \%)$ & $8(42.1 \%)$ & $19(100 \%)$ & \\
Total & $97(56.1 \%)$ & $76(43.9 \%)$ & $173(100 \%)$ & 0.865 \\
Stigmatization & & & & \\
High & $51(51.5 \%)$ & $48(48.5 \%)$ & $99(100 \%)$ & \\
Low & $46(62.2 \%)$ & $28(37.8 \%)$ & $74(100 \%)$ & 0.205 \\
Total & $\mathbf{9 7 ( 5 6 . 1 \% )}$ & $76(43.9 \%)$ & $173(100 \%)$ & \\
\hline & & & & \\
\hline
\end{tabular}


The majority $55.8 \%$ (86) of the participants who were found to have positive religious belief were adherent to cART in this study. Despite having positive religious beliefs $44.2 \%$ (68) were non adherent to cART. However, adherence to cART was found to be independent of religious belief (p-value of 0.865). Among respondents that were non adherent to cART 63\% (48) had high level of stigmatization and $37 \%$ (28) had low level stigmatization. Respondents who were adherent to cART 53\% (51) had high level of stigmatization. The association of adherence to cART and stigma was found to be independent (p-value of 0.205).

In order to test the impact of the predictor variables (knowledge of HIV and disease progression, social support, religious beliefs, stigmatization, Being reminded to take the cART drugs, side effects of drugs and understanding of reason for taking cART) on the outcome variable (adherence to cART), a binary logistic regression was done.

\section{2) Binary logistic regression}

The regression model was statistically significant as a whole $\left(X^{2}=26.621, \mathrm{p}=\right.$ 0.002 ) and could account for $19.1 \%$, variation in the outcome variable, making it a weak model. In terms of prediction power, the model could predict non adherence with $59.2 \%$ accuracy and adherence with an accuracy of $75.3 \%$. As a whole, the model had an overall prediction accuracy of $68.2 \%$.

Further data analysis with the binary logistic regression found that, inside the equation changes in knowledge levels from low to high, social support from inadequate to adequate, religious beliefs from negative to positive, and stigma from low to high did not contribute significantly to the model. Being reminded to take cART variable as a whole was impacted significantly on the model $(p=0.016)$, and changes in reminders to take treatment from never to sometimes contributed significantly but reduced the odds of adherence by $68.5 \%$ ( $\mathrm{p}=0.004$, odds ratio $=0.315$ ). While changes in reminder from sometimes to always reduced the odds of adherence by $40.5 \%$ though the impact was not statistically significant ( $\mathrm{p}$ $=0.27$, odds ratio $=0.505$ ).

The overall impact of the understanding variable was statistically significant $(p=0.011)$. Changes in understanding from not at all to sometimes raised the odds of adherence by $93.5 \%$ but did not impact significantly on the model ( $\mathrm{p}=$ 0.578 , odds ratio $=1.935$ ) while changes from sometimes to very much raised the odds of adherence by $498.2 \%$ although the impact was not statistically significant $(p=0.12$, odds ratio $=5.982)$. Changes in the experience of side effects of the drugs from no to yes contributed significantly to the model and reduced the odds of adherence to $41.2 \%$ ( $p=0.047$, odds ratio $=0.412$ ).

\section{Discussions of Findings}

\subsection{Socio-Demographic Characteristics of the Sample}

The social demographic characteristics showed that $50.9 \%$ (88) of the respondents interviewed were female. The slightly high proportion of female respondents may be due to older male partners infecting them in contexts of poverty, 
manipulation and exploitation without condom use [30]. To support this view the current study found that $1.7 \%$ (3) of the HIV positive adolescents respondents got the infection from being raped by males in their home set ups. Men in such relationships often have multiple sexual partners and acquire sexually transmitted infections, which they pass on to adolescent females [23]. The high female rates may be related to unprotected heterosexual relations with multiple partners [31]. An additional factor contributing to the sex imbalance in adolescent HIV prevalence may be the higher risk of AIDS-related mortality in male adolescents compared to females, which may be related to the lower proportion of males who receive cART [10].

Similar to the current study, higher rates of HIV among females than males have been reported in sub-Saharan Africa [32]. According to UNICEF reports, females aged 15 to 17 years in the sub-Saharan region have up to four times higher prevalence rates of HIV reported than among their male counterparts [33]. The similar high prevalence among females may be due to similar inter-cultural belief systems and practices which promote HIV transmission to the girl child. The practices that include high cases of early marriages to female adolescents and adult HIV infected men practicing sexual intercourse rituals with minor girl to try and cure the infection [3].

Contrary to findings in the current study, the highest HIV prevalence rate in the United States and parts of Europe was reported among males [34]. In the developed settings the prevalence among male adolescents could be higher because of injectable illicit drugs abuse using same needle by several group members. The use of the same needle among several adolescents within the same gang, could contribute to high HIV rates among males [35].

The study also revealed that more than half $(53.2 \%$ (92)) of the respondents were within the age group $16-17$ years. This statistics suggest that most of the perinatally infected adolescents are now living longer attributed to improved HIV/AIDS care services. Most $(52.6 \%$ (91)) of the respondents had attained secondary school education. This is contrary to the beliefs that HIV diagnosis is a death sentence and there was no need to pursue career or educational goals [36]. Literature has shown that with the advances in effectiveness and availability of cART, HIV infected children are now surviving to adolescence and emerging as a new group in the global HIV/AIDS pandemic [24]. It has been also cited that despite the fact that HIV positive adolescents find it a challenge living with the HIV, they have remained positive and focused with their education [24].

\subsection{Adherence to cART}

Respondents in the current study (44\% (76)) were found to be non adherent to their prescribed cART (Figure 1). It must be emphasized that adherence to cART is critical for successful viral suppression because non adherence could lead to an increase in HIV viremia, risk of treatment failure, and accumulating resistance mutations [37]. The $44 \%$ non adherence rate is very alarming and 
pauses as a threat to the maintenance of future cART regimen among HIV positive adolescents due to possible occurrences of related drug resistance and virologic failure.

Similar to the current study, in Thailand slightly higher (48.4\%) suboptimal adherence to cART among adolescents was reported [24]. Despite difference in geographical locations it has been noted that there could be some similar characteristics which exist and affecting the pattern of cART adherence among adolescents [38]. It could be said that non adherence to cART is becoming a global phenomenon and collective strategic efforts need to be developed to prevent the risk of development of treatment failure. Several studies in sub-Saharan Africa have also reported poor adherence among adolescents which is of significant concern given the limited cART options available and the risk of drug resistance [38] [39]. Non-adherence to cART has been perceived as an emerging public health concern [39].

Among the respondents that were found to be non adherent to cART, slightly above half 51\% (39) were males. These findings in the current study confirms the growing body of evidence indicating that male adolescents face challenges in accessing HIV services more often than their female counterparts. The males also experience worse treatment outcomes, including high mortality [40]. With these challenges among male adolescents it is likely that they may not adhere to their cART. Elsewhere evidence has also shown sex-specific differences outcomes that male adolescents have worse adherence compared to their female counterparts [41]. Globally more emphasis has been placed on protection of the girl child and as a result the male child seems to be disadvantaged much more in health related matters [30]. However, it was found that gender was independent of adherence to cART ( $\mathrm{p}$-value of 0.760 ). This could be attributed to the fact that the pathogenesis of HIV is similar despite the person's gender.

The current study found that majority of the respondents $57 \%$ (43) who where non adherent were aged between 16 and 17 years old. This coincides with the age when adolescents are transitioned from pediatrics to adults HIV care. The higher non adherence to cART occurring during this period could be attributed to some challenges associated with implementation of transitioning process of care. A study in West Africa observed that transitioning of care from pediatrics to adult should be a gradual process according to the maturity of the adolescents with evidence of psychological stability and readiness of independent care [30].

On a contrary to the current study, another Zambian study found that there was suboptimal adherence to cART mainly due to increase in age [18]. This could have been that as adolescents increase in age, they are expected to take up more gender roles and responsibilities in society. It has been documented that the successful fulfillment of new gender roles and responsibilities come with challenges that may disrupt the routine daily activities including medication timings [42].

Adherence to cART was found to be independent (p-value 0.428) of the age of the adolescent in the current study. The adolescents' age did not significantly in- 
fluence adherence and there was a minimal difference in the pattern of adherence to cART between older and younger adolescents. It can be argued that despite the age of the adolescents they experience similar influences of adherence to cART. Although some studies have shown high rates of cART adherence among HIV-infected adolescents, a lower rate of adherence among HIV-infected adolescents has been reported more often, and expectations have been that adherence will diminish over time [24] [42]. More cohort studies need to be conducted to identify the non enhancers of adherence to cART.

\subsection{Knowledge of HIV and Disease Progression}

In the current study, majority $(59.5 \%(10))$ of the respondents were found to have low level knowledge about HIV and disease progression (Figure 2). Earlier CDC had highlighted that low level of knowledge among adolescents could be due to limited access to health information and little sources of information about HIV and disease progression [35]. The health information could easily be shared in adolescents-friendly health corners, which according to Zambian consolidated treatment and prevention guidelines are very limited in most health care centers [12]. Limited adolescent-friendly corners may therefore contribute to low level of knowledge about HIV and disease progression. Efforts to improve knowledge and maintain adherence to cART, have been noted where adolescents training curriculum have been developed by the Zambian Ministry of Health in collaboration with International Center for AIDS Care and Treatment Programs [12]. This is aimed at empowering adolescents and caregivers with youth-friendly HIV care information. Also caregiver support was mentioned [29] as a major facilitator of adherence among adolescents with a chronic disease, but this needs a lot of education on disease etiology among all those in this age group. This initiative could also mean that most interventions to promote adherence to cART among adolescent patients can easily be universally applied to enhance of knowledge on HIV and disease progression.

The findings in the current study are consistent with CSO report were it was also reported that the comprehensive HIV knowledge among adolescents was also very low that is, $39 \%$ and $45 \%$ for females and males respectively [3]. It could be argued that adolescents depend on healthcare providers for information about their health and cannot independently seek to acquire more knowledge about their health. Therefore health care providers must be proactively providing health related massages for the adolescents to acquire more knowledge about HIV and the disease progression.

Contrary to the current study, conducted in Zambia found $56.7 \%$ of the adolescents had knowledge about their HIV status [43]. Despite the reported knowledge it was found that adolescents had challenges with maintaining the desired $95 \%$ adherence threshold. The $95 \%$ adherence to cART has been strongly linked to viral suppression in most studies.

Similar to the current study UNICEF reported that the overall level of knowledge of HIV and AIDS remained very low among older adolescents aged 15 - 19 
[23]. Interestingly to note that similar findings were reported in Ghana that $47 \%$ of adolescents on cART had no knowledge about the HIV disease and its progression [44]. These similarities could mean that adolescents have common interests and aspiration despite geographical variation. It could also mean that there is very little or no impact on the current trends of practice to enhance knowledge on HIV and disease progression. This is because low level of knowledge has kept on being reported hence the need for studies on impact assessment on the HIV care models implementation.

However, when adherence to cART was associated with knowledge of HIV and disease progression in the current study, the relationship was found to be independent ( $\mathrm{p}$-value of 0.585 ) (Table 1). Having Knowledge about HIV and disease progression alone cannot prompt improved adherence to cART. This is evident in some more recent studies were despite high knowledge among HIV positive adolescents report [24] [44] [45], it was found that they were non adherence to cART. It could be argued that acquisition of high level knowledge alone is not enough to promote adherence to cART among HIV positive adolescents. A holistic approach in devising interventions must be adapted as the most accurate measure to promoting adherence to cART among HIV positive adolescents. A combination of personal, interpersonal, community and societal interventions as prescribed by social ecological model levels could be the most effective approach to improving adherence to cART among adolescents.

The current study reported that understanding the reason why the respondents were taking cART was found to be strongly dependent (p-value 0.006) with adherence to cART. Having understanding could mean having the ability to interpret and explain information about HIV and being able to estimate and anticipate undesired outcomes when there is poor adherence to cART. It is expected that those respondents who had understanding of the reason they were taking cART, also knew the consequences of non adherence. Therefore, they were compelled to be adherent to their cART to prevent disease progression and drug resistance.

Similar to the current study some studies elsewhere have also shown that having understanding of the benefits for taking cART could have a positive influence on cART adherence among HIV positive adolescents [46]. These finding suggest that promotion of adherence to cART, requires deeper level of knowledge about HIV and disease progression.

The current study established that $58 \%$ (56) of the respondents (Table 2) who were adherent to cART had low level knowledge. This could mean that the adolescents who acquire more knowledge feel that it is enough to prevent the disease progression. This is evident by higher pattern of adherence to cART by the respondents who had low knowledge 62\% (47). The respondents who had low knowledge of HIV and disease progression had more than half times chance more likely to be adherent to cART than those who had high level of knowledge about HIV and disease progression (Table 2). This could be due to the caregiver's strictness in ensuring that the adolescents are adherent to cART who may 
Table 2. Binary logistic regression output-variables in the equation.

\begin{tabular}{|c|c|c|c|c|}
\hline \multirow{2}{*}{ Variable } & \multirow{2}{*}{ p-Value } & \multirow{2}{*}{ Odds Ratio } & \multicolumn{2}{|c|}{ 95\% C.I. for Odds Ratio (1.0 ref) } \\
\hline & & & Lower & Upper \\
\hline \multicolumn{5}{|l|}{ Knowledge (1) } \\
\hline High & 0.375 & 0.723 & 0.353 & 1.481 \\
\hline Low & & 0.839 & 0.456 & 1.557 \\
\hline \multicolumn{5}{|l|}{ Social Support (1) } \\
\hline Adequate & 0.369 & 1.993 & 0.442 & 8.983 \\
\hline Inadequate & & 0.158 & 0.341 & 1.346 \\
\hline \multicolumn{5}{|l|}{ Religious Beliefs (1) } \\
\hline Positive & 0.818 & 0.885 & 0.311 & 2.514 \\
\hline Negative & & 0.029 & 0.452 & 1.232 \\
\hline \multicolumn{5}{|l|}{ Stigma (1) } \\
\hline High & 0.228 & 0.644 & 0.315 & 1.316 \\
\hline Low & & 0.205 & 0.562 & 1.785 \\
\hline Reminding & 0.016 & & & \\
\hline Reminding (1) & 0.004 & 0.315 & 0.144 & 0.691 \\
\hline Reminding (2) & 0.27 & 0.505 & 0.15 & 1.698 \\
\hline Side Effects (1) & 0.047 & 0.412 & 0.172 & 0.987 \\
\hline Understand & 0.011 & & & \\
\hline Having Understanding (1) & 0.578 & 1.935 & 0.189 & 19.83 \\
\hline No Understanding (2) & 0.12 & 5.982 & 0.629 & 56.895 \\
\hline Constant & 0.769 & 0.627 & & \\
\hline
\end{tabular}

have low knowledge of HIV and disease progression. The adolescents who had low level of knowledge could also be depending on their buddies and healthcare providers for the instructions and care hence the higher rate of adherence to cART among those reported to have low level knowledge.

\subsection{Social Cultural Factors}

\subsubsection{Social Support System}

The majority (94.8\% (164)) of the respondents reported to had adequate social support (Figure 3). These results could be that the current study site had better resources than many clinics in resource limited settings. The site also had a supportive environment that equipped adolescents with the appropriate skills to enable them to face the challenges of adolescence. This may in part explain the documented adequate social support here. Similarly to the current study some scholars in earlier studies elsewhere also reported adequate social support. It was found that adequate social support improved patient's ability to be adherent to cART [47] [48] [49]. Without physical and psychological support, it would be very difficult for the adolescents to access healthcare services. The reported ade- 
quate social support could also mean that the adolescents with HIV are living in conducive and supportive environments. While the issues of poor social support exist commonly in resource-rich settings, they may be even more prevalent in resource-limited settings and affecting adherence to cART [50]. On the contrary most studies reports indicates that social support is adequate in both resource-rich and resource-limited settings.

In the current study it was found that adherence to cART and social support were independent. Social support when associated with adherence to cART among HIV positive adolescents, there was no significant finding ( $\mathrm{p}$-value of 0.158). This could be explained by the statistic that $92 \%$ (70) of the respondents in the current study who were non adherent to cART were reported to have adequate social support (Table 1). It can be concluded that non adherence to cART by adolescents could be contributed by personal rather than interpersonal related aspects. This is deduced because being reminded to taking medications in the current study was found to be dependent of adherence to cART ( $p$-value of 0.006). A binary logistic regression was performed to ascertain dependence of being reminded to taking the cART and adherence to cART. The logistic regression model was statistically significant (p-value 0.0004 ; OR 0.315 ). The HIV positive adolescents need to be encouraged by others around them to continue with cART and all the clinical follow ups as scheduled for the success of treatment.

Unfortunately $41 \%$ (71) respondents had nobody to go to the hospital to collect their CART on their behalf. When adolescents miss their hospital appointments they could easily run out of drugs. Missing hospital appointments could interference with cART adherence hence drug sub-therapeutic serum concentration levels leading to viral mutations and viral resistance [51]. Essentially if a patient has ever missed a refill, he or she is more likely to miss some doses suggesting an increased risk of non adherence to cART even in the absence of ongoing refill problems [52].

Furthermore $80.3 \%$ (139) of the respondents reported that when they missed the hospital appointments, no follow up was made to establish the reason by the healthcare providers. In most healthcare facilities in resource-limited settings, the health worker to patient ratios are overwhelming. The health workers may not easily remember which patients are missing their appointments due to large patient populations seen. The patient missed appointment tracking system would be the most ideal when dealing with large numbers of adolescents patients. Healthcare providers need to have an established patient appointment tracking system to easily identify adolescents who could be missing out on drug refills due to low or no family support. Those missing appointments $27.7 \%$ (48) reported finding it difficult sometimes to access healthcare. The fact that some adolescents find it difficult to access care to pick up cART medications, suggests existence of structural barriers. Therefore, practical assistance to adolescents makes them feel cared for when given help such as financial support for transportation and easy access to get some drug refills and clinical follow up appointments. Support from parents (both financial and social) was highly rated 
by respondents in a study as positive [53]. The study suggested that reinforcement of family closeness, cohesiveness, and problem-solving skills with adolescents could help the adolescents to complete the tasks related to treatment successfully on the daily basis.

Evidence from one study findings agree that adolescents with extensive supportive social networks among healthcare providers, relatives and peers appeared to cope better with psychosocial challenges [54]. The community is supposed to have social network systems in place aimed at identification and offering active support to HIV positive adolescents on cART. This is because adolescence is a critical stage of a persons' transition from childhood to adulthood. Studies have observed that not all adolescents living with HIV were ready to make transfer from children to adult care at the same time [55]. Before any transfer of care, health care workers should take into account the HIV positive adolescents' cognitive and physical development and emotional maturity, their support at home and community.

\subsubsection{Religious Beliefs}

More than half (52.1\% (90)) of the respondents in the current study believed that prayers and anointing oil could cure HIV/AIDS. Religious belief system has a very strong influence on an individual's life and behavior. It has been cited that religion serves important roles in coping, survival and maintaining overall wellbeing within African cultures and communities, especially when diagnosed with a chronic disease like HIV/AIDS that can have a profound effect on physical and mental health [56]. However, spirituality/religion can be problematic to some patients and results in healthcare provision challenges.

Earlier study in Zambia showed that $17 \%$ of participants believed that HIV could be cured by prayers and taking ART [57]. Religious belief system that prayer and anointing oil could heal HIV/AIDS by some groupings seems to be gaining ground among HIV positive adolescents. This could be supported by report in the current study where 9\% (15) of the HIV positive adolescents had at one point abandoned cART in preference of prayers. Researchers suggest that some religious beliefs and doubts about antiretroviral therapy among adolescents may be cultural specific, where faith-based healing is propagated by leaders of these faith communities. It is argued that health-related spiritual beliefs like calling on prayers or a higher power for protection to take control of health is common among patients with life-threatening diseases like cancer, mental illness, and HIV/AIDS [58]. Patients who accept such beliefs will not overtly reject cART when offered, but most often will not adhere to treatment and wait to report miraculous healing. It could be evident by report in the current study where $44.2 \%$ (68) respondents despite having positive religious beliefs were non adherent to cART. This could be that there is a mixed belief system between healing by prayer and cART adherence [58]. However, adherence to cART was found to independent of religious belief ( $\mathrm{p}$-value of 0.865 ).

Elsewhere a study on ARV adherence in Uganda, found that 6 out of 558 
(1.2\%) adolescents discontinued their treatment because they believed that their pastors' prayers had cured them of HIV [59]. Discontinuation of cART risk is gradually being reported and could actualize into health problem. Clergymen claiming healing powers need to start giving HIV clearance certificates following claims of curing HIV after offering prayers. This would protect the patients at risk of discontinuing medical treatment from seeking cure through prayers. These beliefs have already led to some adolescent patients to stopping cART after being promised to be cured of their HIV infection through prayers and anointing oil as evident in the current study report.

\subsubsection{Stigmatization}

In the current study generally it was reported that $57.2 \%$ (99) of the respondents had experienced high level of stigmatization. Despite the wide availability of campaign massages against stigma and discrimination, it's evident that there are socio-cultural misperceptions about the etiology and spread of HIV/AIDS. This could be the reason for the high reported stigma in some parts of sub-Saharan Africa including in the current study.

Similar to the current study, in Eastern Europe and Central Asia a study also reported high level of stigmatization with $61 \%$ HIV positive adolescents [60]. The study also linked stigmatization to non-adherence to cART. However this was contrary to the current study were adherence to cART and stigma was found to be independent ( $\mathrm{p}$-value of 0.205 ). This could also suggest that in a long run stigmatized individual would eventually become non-adherent to their cART. Consistent the current report a study in the USA, also reported that $76 \%$ of adolescents experiencing HIV-related stigma [61]. This impacted negatively on their routine taking of the cART. One study observed that adolescents are often concerned about "feeling normal" and not feeling "different from their peers" [55]. Apart from the inherent difficulty of repeatedly taking medications, adolescents sometimes skip cART doses because drugs are a reminder of the HIV infection that makes them different from others.

Among respondents that were non adherent to cART 63\% (48) had high level of stigmatization (Table 2). According to the WHO report, stigmatization leads to poor management of HIV/AIDS programs and violation of human rights for the HIV positive adolescents. When stigmatization is high, the affected adolescents could easily abandon their cART or even fail to follow up the scheduled clinical visits [15]. This is evident where nearly one third $30.1 \%$ (52) of the respondents reported failing taking their cART because of friends, teachers or other people being around. During social outings away from home such as in camps and sporting activities, the HIV positive adolescents could be uncomfortable to take their antiretroviral drugs resulting in reduction of therapeutic drug levels in their blood to suppress the viral load. This could lead to viral mutation and continued viral multiplication hence increasing the amount of HIV in the blood.

It must be stated that stigmatized individuals are more likely going to suffer social isolation and non adherence to their cART. Non adherence to cART pre- 
vents maximal and durable suppression of HIV replication.

In contrast to the current report, studies in Kenya and Uganda have demonstrated decline in stigmatization among adult patients after a long period on ART [62] [63]. This observation is vital that reduction of stigmatization is a long term gradual process. This is supported by the findings of qualitative studies among people with HIV in Zimbabwe and South Africa, who generally attributed their improved self-image, functioning and wellbeing to the role of antiretroviral treatment [64] [65]. It is expected that after a long time on cART the adolescents would be living quality lives free from opportunistic infections. When the attained quality of life is linked to adherence to cART, stigmatization would not affect the adherence.

\section{Study Limitations}

The study did not explore ways to follow up victims infected with HIV through rape to ensure justice. The study was limited to HIV positive adolescents enrolled at UTH, adult infectious diseases Centre-HIV care and treatment site. However a representative sample size was obtained to allow transferability and generalization of findings to adolescents in general population.

Data was limited to respondents' responses to the questionnaire question items. Hence some experiences of the respondents that led to non adherence to cART were not provided from the responses.

\section{Conclusions}

The purpose of the current study was to determine factors influencing adherence to cART among HIV positive adolescents at Adult Infectious Diseases Center in Lusa$\mathrm{ka}$, Zambia. It was found that there was significantly high level of non-adherence to cART among HIV positive adolescents. The adherence to cART of $95 \%$ or above is necessary for maintaining viral suppression; preservation of health and attainment of physical and psychological wellbeing.

The current study reported there was low level of knowledge and high level of stigmatization among the respondents. However, when predictors variables (level of knowledge of HIV and disease progression, social support, religious beliefs and level of stigmatization) were associated with the outcome variable (adherence to $\mathrm{CART}$ ), they were found to be independent. Therefore, the current study accepted the null hypothesis that there was no relationship between adherence to cART (dependant variable) and Knowledge of cART treatment and disease process and social cultural factors (independent variables) among HIV positive adolescents.

Other factors such as understanding the correct reason for taking cART, experiencing cART side effects, being reminded to taking the drugs and just being satisfied with the care being provided were found to significantly influence adherence to cART. The study findings suggest that once HIV positive adolescents understand the need to take their cART, they would play a pivotal role to sustain 
adherence of $95 \%$ or more to the prescribed cART. This would lead to achievement of the benefits of sustained viral suppression for as long time as possible.

\section{Recommendations}

To address the problem of non-adherence to cART, support by Health care providers is essential until the adolescents' competency and responsibility are assured. Therefore directly observed antiretroviral therapy administration strategy by buddies must be introduced among HIV positive adolescents since being reminded to taking cART was dependant to adherence.

More studies must be conducted in sub-Saharan Africa to inform the development of culturally centered appropriate interventions and strategies to optimize cART adherence among adolescents.

The Individual targeted interventions such as enhancing HIV knowledge to improve understanding of the disease process using electronic animations could be an effective enhancer of adherence to cART among HIV positive adolescents.

More emphasis must be made by promotion of interpersonal focused intervention such as peer group support through sharing experiences hence mitigation of stigmatization and discrimination which was reported to be prevalent among HIV positive adolescents.

The churches association of Zambia (CHAZ) needs to pass a regulatory framework on who should speak about HIV cure among the churches denominations and other religious groupings. This is to prevent some clergymen stopping HIV positive adolescents from taking their prescribed cART and replacing them with anointing oil and prayers. This practice has a great risk of viral mutation and virologic failure related to interruption of prescribed cART.

\section{Acknowledgements}

The author is grateful to the University of Zambia, School of Nursing Sciences for the mentorship, encouragements rendered before and during this research study.

\section{Conflicts of Interest}

The authors declare no conflicts of interest regarding the publication of this paper.

\section{References}

[1] UNAIDS (2011) Opportunity in Crisis: Preventing HIV from Early Adolescence to Young.

[2] UNICEF (2015) Strengthening the Adolescent Component of National HIV Programmes through Country Assessments. UNICEF, New York.

[3] Central Statistical Office (CSO), Ministry of Health (MOH), and ICF International (2014) Zambia Demographic and Health Survey 2013-14. Rockville, Maryland, Central Statistical Office, Ministry of Health, and ICF International, USA. 
[4] Haberer, J.E., Cook, A., Walker, A.S., Ngambi, M., Ferrier, A. and Mulenga, V. (2011) Excellent Adherence to Antiretrovirals in $\mathrm{HIV}^{+}$Zambian Children Is Compromised by Disrupted Routine, HIV Nondisclosure, and Paradoxical Income Effects. PLoS ONE, 6, e18505. https://doi.org/10.1371/journal.pone.0018505

[5] Panel on Antiretroviral Guidelines for Adults and Adolescents (2016) Guidelines for the Use of Antiretroviral Agents in HIV-1-Infected Adults and Adolescents. Department of Health and Human Services. http://www.aidsinfo.nih.gov/ContentFiles/AdultandAdolescentGL.pdf

[6] Agwu, A.L., Bethel, J. and Hightow-Weidman, L.B. (2012) Substantial Multiclass Transmitted Drug Resistance and Drug-Relevant Polymorphisms among Treatment-Naive Behaviorally HIV-Infected Youth. AIDS Patient Care STDS, 4, 193-196. http://www.ncbi.nlm.nih.gov/pubmed/22563607 https://doi.org/10.1089/apc.2011.0420

[7] WHO (2013) Consolidated Guidelines on the Use of Antiretroviral Drugs for Treating and Preventing HIV Infection: Recommendations for a Public Health Approach.

[8] Chkhartishvili, N., Sharvadze, L., Dvali, N., Karchava, M., Rukhadze, N., Lomtadze, M. and Tsertsvadze, T. (2014) Virologic Outcomes of Second-Line Antiretroviral Therapy in Eastern European Country of Georgia. AIDS Research and Therapy, 11, 18. https://doi.org/10.1186/1742-6405-11-18

[9] WHO (2016) Global AIDS Response Progress Reporting (WHO/UNICEF/UNAIDS) and WHO HIV Country Intelligence Tool. Geneva.

[10] UNAIDS (2015) Access to Antiretroviral Therapy in Africa: Status Report on Progress.

[11] Paredes, R., Lalama, C.M. and Ribaudo, H.J. (2010) Pre-Existing Minority Drug-Resistant HIV-1 Variants, Adherence, and Risk of Antiretroviral Treatment Failure. Journal of Infectious Diseases, 201, 662-671.

http://www.ncbi.nlm.nih.gov/entrez/query.fcgi?cmd=Retrieve\&db=PubMed\&dopt= Citation\&list_uids $=20102271$ https://doi.org/10.1086/650543

[12] MoH (2014) Zambia Consolidated Guidelines, for Treatment and Prevention of HIV Infection. Lusaka, Zambia.

[13] CDC (2016) HIV/AIDS Surveillance Report, Vol. 16. US Department of Health and Human Services, Atlanta, Georgia.

[14] Edward, C. and Klatt, M.D. (2016) Pathology of HIV/AIDS Version 27 Edward C. MD Mercer University School of Medicine Savannah, Klatt.

[15] WHO (2013) Report on AIDS Epidemic. www.unaids.org/en/resources/campaigns/globalreport2013/globalreport

[16] Buchanan, A.L., Montepiedra, G., Sirois, P.A., Kammerer, B., Garvie, P.A. and Storm, D.S. (2012) Barriers to Medication Adherence in HIV-Infected Children and Youth Based on Self- and Caregiver Report. Pediatrics, 129, e1244-e1251. https://doi.org/10.1542/peds.2011-1740

[17] Seu, L., Mulenga, L.B. and Siwingwa, M. (2015) Characterization of HIV Drug Resistance Mutations among Patients Failing First-Line Antiretroviral Therapy from a Tertiary Referral Center, Lusaka, Zambia.

[18] Stalter, R.M., Katayamoyo, P., Packer, C. and Banda, H. (2016) Transitioning to Second-Line Antiretroviral Therapy among Adolescents. Copperbelt Province, Zambia. 
[19] Ryscavage, P. and Anderson, E.J. (2011) Clinical Outcomes of Adolescents and Young Adults in Adult HIV Care. Journal of Acquired Immune Deficiency Syndromes, 58, 193-197. http://www.ncbi.nlm.nih.gov/pubmed/21826014 https://doi.org/10.1097/QAI.0B013E31822D7564

[20] Melsew, Y.A., Terefe, M.W., Tessema, G.A. and Ayele, T.A. (2013) Rate of Immunological Failure and Its Predictors among Patients on Highly Active Antiretroviral Therapy at Debremarkos Hospital, Northwest Ethiopia. Journal of AIDS and Clinical Research, 4, 211.

[21] Baggini, J. (2011) To Debate Religion, We Must First Find Out What People Believe. The Guardian.

http://www.guardian.co.uk/commentisfree/belief/2011/nov/14/debate-religion-what -people-believe

[22] Snyder, K. (2014) Preliminary Results from Hlanganani (Coming Together): A Structured Support Group for HIV-Infected Adolescents Piloted in Cape Town, South Africa.

[23] UNICEF (2012) Progress for Children: A Report Card on Adolescents. No. 10, New York.

[24] Xu, L., Munir, K., Kanabkaew, C. and Le Coeur, S. (2017) Factors Influencing Antiretroviral Treatment Suboptimal Adherence among Perinatally HIV-Infected Adolescents in Thailand. PLOS ONE, 12, e0172392.

https://doi.org/10.1371/journal.pone.0172392

[25] Sallis, J. and Owen, N. (2002) Ecological Models of Health Behavior. In: Glanz, K., Ed., Health Behavior and Health Education: Theory, Research and Practice, 3rd Edition, John Wiley \& Sons, New York, 462-484.

[26] Polit, D.F. and Hungler, B.P. (2013) Essentials of Nursing Research: Methods, Appraisal, and Utilization. 8th Edition, Lippincott Williams and Wilkins, Philadelphia.

[27] Krejcie, R.V. and Morgan, D.W. (1970) Determining Sample Size for Research Activities. Educational and Psychological Measurements, 30, 607-610. https://doi.org/10.1177/001316447003000308

[28] WMA (2008) Declaration of Helsinki: Ethical Principles for Medical Research Involving Human Subjects. Helsinki, Finland.

[29] Mburu, G., Hodgson, I. and Kalibala, S. (2014) Adolescent HIV Disclosure in Zambia: Barriers, Facilitators and Outcomes. Journal of the International AIDS Society, 17, 18866. https://doi.org/10.7448/IAS.17.1.18866

[30] Adejumo, O.A., Malee, K.M., Ryscavage, P., Hunter, S.J. and Taiwo, B.O. (2015) Contemporary Issues on the Epidemiology and Antiretroviral Adherence of HIV-Infected Adolescents in Sub-Saharan Africa: A Narrative Review. Journal of the International AIDS Society, 18, 20049. https://doi.org/10.7448/IAS.18.1.20049

[31] Idele, P., Gillespie, A., Porth, T. and Suzuki, C. (2014) Epidemiology of HIV and AIDS among Adolescents, Current Status, Inequities, and Data Gaps. Journal of Acquired Immune Deficiency Syndromes, 66, S144-S153. https://doi.org/10.1097/QAI.0000000000000176

[32] Parienti, J.J. (2014) The Case of Adherence in the Youth: Rebel without a Cause? AIDS, 28, 1983-1985. https://doi.org/10.1097/QAD.0000000000000317

[33] UNAIDS (2014) 20th International AIDS Conference. Melbourne, Australia. Global Fact Sheet: HIV/AIDS.

http://www.unaids.org/en/resources/campaigns/globalreport2013/factsheet

[34] UNICEF (2014) Preventing HIV Infection among Adolescents and Young People. 
Nairobi. http://www.unicef.org/esaro/5482_HIV_prevention.html

[35] Centers for Disease Control and Prevention (2014) HIV in the United States: The Stages of Care-CDC Fact Sheet.

http://www.cdc.gov/nchhstp/newsroom/docs/HIV-Stages-of-Care-Factsheet-508.pd $\mathrm{f}$

[36] Baumgartner, L.M. (2012) The Perceived Effects of the Socialcultural Context on HIV/AIDS indentity Incorporation. The Qualitative Report, 17, 1-21.

[37] Boussari, O., Subtil, F., Genolini, C., Bastard, M., Iwaz, J. and Fonton, N. (2015) Impact of Variability in Adherence to HIV Antiretroviral Therapy on the Immunovirological Response and Mortality. BMC Medical Research Methodology, 15, 10. https://doi.org/10.1186/1471-2288-15-10

[38] Nglazi, M.D., Kranzer, K., Holele, P., Kaplan, R., Mark, D. and Jaspan, H. (2012) Treatment Outcomes in HIV-Infected Adolescents Attending A Community-Based Antiretroviral Therapy Clinic in South Africa. BMC Infectious Diseases, 12, 21. https://doi.org/10.1186/1471-2334-12-21

[39] Muri, L., Gamell, L., Ntamatungiro, A., Glass, T.R. and Luwanda, L.B. (2016) Development of HIV Drug Resistance and Therapeutic Failure in Children and Adolescents in Rural Tanzania: An Emerging Public Health Concern. AIDS, 31, 61-70. https://doi.org/10.1097/QAD.0000000000001273

[40] Druyts, E., Dybul, M. and Kanters, S. (2013) Male Sex and the Risk of Mortality among Individuals Enrolled in Antiretroviral Therapy Programs in Africa: A Systematic Review and Meta-Analysis. AIDS, 27, 417-425. https://doi.org/10.1097/QAD.0b013e328359b89b

[41] Lowenthal, E.D., Ellenberg, J.H. and Machine, E. (2013) Association between Efavirenz-Based Antiretroviral Regimens and Virologic Failure in HIV Infected Children. JAMA, 309, 1803-1809. https://doi.org/10.1001/jama.2013.3710

[42] Evans, D., Menezes, C. and Mahomed, K. (2013) Treatment Outcomes of HIV-Infected Adolescents Attending Public-Sector HIV Clinics across Gauteng and Mpumalanga, South Africa. AIDS Research and HumanRetroviruses, 29, 892-900. https://doi.org/10.1089/aid.2012.0215

[43] Mweemba, M., Musheke, M.M., Michelo, C., Halwiindi, H., Mweemba, O. and Zulu, J.M. (2015) When Am I Going to Stop Taking the Drug? Enablers, Barriers and Processes of Disclosure of HIV Status by Caregivers to Adolescents in a Rural District in Zambia. BMC Public Health, 15, 1028.

https://doi.org/10.1186/s12889-015-2372-3

[44] Kenu, E., Adjoa, O.A., Nuamah, G., Brefo, A., Sam, M. and Lartey, M. (2014) Knowledge and Disclosure of HIV Status among Adolescents and Young Adults Attending an Adolescent HIV Clinic in Accra, Ghana. BMC Research Notes, 7, 844. https://doi.org/10.1186/1756-0500-7-844

[45] Hornschuh, S., Dietrich, J.J., Tshabalala, C. and Laher, F. (2017) Antiretroviral Treatment Adherence: Knowledge and Experiences among Adolescents and Young Adults in Soweto, South Africa. AIDS Research and Treatment, 2017, Article ID: 5192516. https://doi.org/10.1155/2017/5192516

[46] Agwu, A.L. and Fairlie, L. (2013) Antiretroviral Treatment, Management Challenges and Outcomes in Perinatally HIV-Infected Adolescents. Journal of the International AIDS Society, 16, 18579. https://doi.org/10.7448/IAS.16.1.18579

[47] WHO (2011) Guideline on HIV Disclosure Counselling for Children up to 12 Years of Age, in HIV/AIDS Programme. Genevia, Switzerland.

[48] Lee, S., Yamazaki, M., Harris, D.R., Harper, G.W. and Ellen, J. (2015) Social Support 
and HIV-Status Disclosure to Friends and Family: Implications for HIV-Positive Youth. The Journal of Adolescent Health, 57, 73-80.

https://doi.org/10.1016/j.jadohealth.2015.03.002

[49] Kahana, S.Y., Rohan, J., Allison, S., Frazier, T.W. and Drotar, D. (2013) A Meta-Analysis of Adherence to Antiretroviral Therapy and Virologic Responses in HIV-Infected Children, Adolescents, and Young Adults. AIDS and Behavior, 17, 41-60. https://doi.org/10.1007/s10461-012-0159-4

[50] Bygrave, H., Mtangirwa, J., Ncube, K., Ford, N., Kranzer, K. and Munyaradzi, D. (2012) Antiretroviral Therapy Outcomes among Adolescents and Youth in Rural Zimbabwe. PLoS ONE, 7, e52856. https://doi.org/10.1371/journal.pone.0052856

[51] WHO (2014) Health for the World's Adolescents. http://apps.who.int/adolescent/second-decade/

[52] Ndiaye, M., Nyasulu, P., Nguyen, H., Lowenthal, E.D., Gross, R., Mills, E.J. and Nachega, J.B. (2013) Risk Factors for Suboptimal Antiretroviral Therapy Adherence in HIV-Infected Adolescents in Gaborone. A Pilot Cross-Sectional Study, Botswana.

[53] Taddeo, D., Egedy, M. and Frappier, J. (2013) Adherence to Treatment in Adolescence. Journal of Paediatrics and Child Health, 13, 19-24.

https://doi.org/10.1093/pch/13.1.19

[54] Peterson, K., Togun, T., Klis, S., Menten, J. and Colebunders, R. (2012) Depression and Posttraumatic Stress Disorder among HIV-Infected Gambians on Antiretroviral Therapy. AIDS Patient Care STDs, 26, 589-596. https://doi.org/10.1089/apc.2012.0089

[55] Fetzer, B.C., Mupenda, B., Lusiama, J., Kitetele, F., Golin, C. and Behets, F. (2011) Barriers to and Facilitators of Adherence to Pediatric Antiretroviral Therapy in a Sub-Saharan Setting: Insights from a Qualitative Study. AIDS Patient Care STDs, 25, 611-621. https://doi.org/10.1089/apc.2011.0083

[56] Arrey, A.E., Bilsen, J., Lacor, P. and Deschepper, R. (2016) Spirituality/Religiosity: A Cultural and Psychological Resource among Sub-Saharan African Migrant Women with HIV/AIDS in Belgium. PLOS ONE, 11, e0159488.

https://doi.org/10.1371/journal.pone.0159488

[57] Nozaki, I., Kuriyama, M., Manyepa, P., Zyambo, M.K., Kakimoto, K. and Bärnighausen, T. (2013) False Beliefs About ART Effectiveness, Side Effects and the Consequences of Non-Retention and Non-Adherence among ART Patients in Livingstone, Zambia. AIDS and Behavior, 17, 122-126. https://doi.org/10.1007/s10461-012-0221-2

[58] Kremer, H., Ironson, G. and Porr, M. (2016) Spiritual and Mind-Body Beliefs as Barriers and Motivators to HIV Treatment Decision-Making and Medication Adherence? A Qualitative Study. AIDS Patient Care STDS, 23, 127-134. https://doi.org/10.1089/apc.2008.0131

[59] Wanyama, J., Castelnuovo, B., Wandera, B., Mwebaze, P., Kambugu, A., Bangsberg, D.R. and Kamya, M.R. (2013) Belief in Divine Healing Can Be a Barrier to Antiretroviral Therapy Adherence. Kampala, Uganda.

[60] Katz, I.T., Ryu, A.E., Onuegbu, A.G., Psaros, C., Weiser, S.D., Bangsberg, D.R. and Tsai, A.C. (2013) Impact of HIV-Related Stigma on Treatment Adherence: Systematic Review and Meta-Synthesis. Journal of the International AIDS Society, 16, 18640. https://doi.org/10.7448/IAS.16.3.18640

[61] Feldman, B.J., Rao, D., Fredericksen, R.J., Crane, P.K., Simoni, J.M., Kitahata, M.M. and Crane, H.M. (2012) A Structural Equation Model of HIV-Related Stigma, Depressive Symptoms, and Medication Adherence. AIDS and Behavior, 16, 711-716. 
https://doi.org/10.1007/s10461-011-9915-0

[62] Kaai, S., Bullock, S., Sarna, A., Chersich, M., Luchters, S. and Geibel, S. (2012) Perceived Stigma among Patients Receiving Antiretroviral Treatment: A Prospective Randomised Trial Comparing an m-DOT Strategy with Standard-of-Care in Kenya. SAHARA J, 7, 62-70.

https://doi.org/10.1080/17290376.2010.9724958

[63] Tsai, A.C., Bangsberg, D.R., Bwana, M., Haberer, J.E., Frongillo, E.A. and Muzoora, C. (2013) How Does Antiretroviral Treatment Attenuate the Stigma of HIV? Evidence from a Cohort Study in Rural Uganda. AIDS and Behavior, 17, 2725-2731. https://doi.org/10.1007/s10461-013-0503-3

[64] Campbell, C., Skovdal, M., Madanhire, C., Mugurungi, O., Gregson, S. and Nyamukapa, C. (2011) We, the AIDS People: How Antiretroviral Therapy Enables Zimbabweans Living with HIV/AIDS to Cope with Stigma. American Public Health Association, 101, 1004-1010. https://doi.org/10.2105/AJPH.2010.202838

[65] Zuch, M. and Lurie, M. (2012) A Virus and Nothing Else: The Effect of ART on HIV-Related Stigma in Rural South Africa. AIDS and Behavior, 16, 564-570.

https://doi.org/10.1007/s10461-011-0089-6 